\title{
Superconducting Analogue of the Parafermion Fractional Quantum Hall States
}

\begin{abstract}
Abolhassan Vaezi*
Department of Physics, Cornell University, Ithaca, New York 14853, USA

(Received 27 August 2013; revised manuscript received 3 November 2013; published 15 July 2014)

Read-Rezayi $Z_{k}$ parafermion wave functions describe $\nu=2+(k / k M+2)$ fractional quantum Hall $(\mathrm{FQH})$ states. These states support non-Abelian excitations from which protected quantum gates can be designed. However, there is no experimental evidence for these non-Abelian anyons to date. In this paper, we study the $\nu=2 / k \mathrm{FQH}$-superconductor heterostructure and find the superconducting analogue of the $Z_{k}$ parafermion $\mathrm{FQH}$ state. Our main tool is the mapping of the $\mathrm{FQH}$ into coupled one-dimensional chains, each with a pair of counterpropagating modes. We show that by inducing intrachain pairing and charge preserving backscattering with identical couplings, the one-dimensional chains flow into gapless $Z_{k}$ parafermions when $k<4$. By studying the effect of interchain coupling, we show that every parafermion mode becomes massive except for the two outermost ones. Thus, we achieve a fractional topological superconductor whose chiral edge state is described by a $Z_{k}$ parafermion conformal field theory. For instance, we find that a $\nu=2 / 3 \mathrm{FQH}$ in proximity to a superconductor produces a $Z_{3}$ parafermion superconducting state. This state is topologically indistinguishable from the non-Abelian part of the $\nu=12 / 5$ Read-Rezayi state. Both of these systems can host Fibonacci anyons capable of performing universal quantum computation through braiding operations.
\end{abstract}

DOI: 10.1103/PhysRevX.4.031009

\section{INTRODUCTION}

There has been a surge of interest in searching for nonAbelian anyons (non-Abelions) [1] in the topological states of matter during the past few years [2-9]. The non-Abelian states are suitable platforms to perform topological quantum computation via braiding their non-Abelions. The simplest non-Abelian state is the Pfaffian wave function that was proposed by Moore and Read to explain the $\nu=5 / 2$ fractional quantum Hall $(\mathrm{FQH})$ plateau [5]. Later, Read and Rezayi generalized Pfaffian to $Z_{k}$ parafermion wave functions as the ground state of $\nu=2+(k / k M+2) \mathrm{FQH}$ states, where $M$ is an even (odd) integer for bosons (fermions) [10]. The neutral sector of the edge state can be obtained by computing the correlation functions of an $S U(2)_{k} / U(1)$ conformal field theory (CFT) known as a $Z_{k}$ parafermion CFT $[1,11]$. These states are believed to have non-Abelian excitations $[1,10]$. Unfortunately, the experimental search for detecting non-Abelian excitations in the $\mathrm{FQH}$ states has failed so far. In this paper, we propose another venue to search for $Z_{k}$ parafermion states and show that these exotic states emerge in an FQH-superconductor heterostructure.

\footnotetext{
*vaezi@cornell.edu
}

Published by the American Physical Society under the terms of the Creative Commons Attribution 3.0 License. Further distribution of this work must maintain attribution to the author(s) and the published article's title, journal citation, and DOI.
Subject Areas: Statistical Physics,

Strongly Correlated Materials,

Topological Insulators

The simplest non-Abelian quasiparticle, the Ising anyon, was conjectured to be found in the $\nu_{c}=5 / 2$ Pfaffian FQH state by Moore and Read [5]. This state corresponds to $Z_{2}$ Read-Rezayi parafermions with $M=1$. It was shown later that Majorana fermions can be observed in the superconducting vortices in the weak-pairing phase of the $p_{x}+i p_{y}$ superconductors as well $[12,13]$, and these two seemingly different states have identical topological order. This $p_{x}+i p_{y}$ was the first known superconducting state that supports non-Abelions expected in FQH states.

The most interesting state among the Read-Rezayi wave functions is the $Z_{3}$ parafermion state that describes the ground state of the $\nu=12 / 5$ and $\nu=13 / 5 \mathrm{FQH}$ states $[1,10]$. This state supports the Fibonacci anyon, a nonAbelion that has $d_{F}=(1+\sqrt{5} / 2)$ quantum dimension and can perform universal quantum computation; i.e., all the quantum gates can be designed and measured by braiding Fibonacci anyons [1,14-16]. The quantum dimension of Fibonacci anyons can be understood by counting the number of degenerate ground states at the presence of $n$ Fibonacci-anyon excitations. For that purpose, we need to consider their fusion algebra: $\tau \times \tau \sim 1+\tau$, which means a state with $n$ Fibonacci anyons can be mapped into the superposition of states with two and one fewer Fibonacci anyons, respectively. Thus, the ground-state degeneracy in the presence of $n$ Fibonacci-anyon excitations $G(n)$ satisfies the Fibonacci recursion relation, i.e., $G(n)=$ $G(n-1)+G(n-2)$. At the large $n$ limit, ground-state degeneracy grows as $\log G(n) \sim n \log d_{F}+\cdots$. 
Achieving the two-dimensional (2D) superconducting analogue of the Read-Rezayi parafermion states requires answering two major steps: First, in what system can we observe parafermions [17-20] as the physical degree of freedom? Second, how can we condense such quasiparticles? Parafermions are the generalization of the Majorana fermion, sometimes called fractionalized Majorana fermions. Consider the $\chi_{i}$ parafermion operator at site $i$. A $Z_{k}$ parafermion operator satisfies $\chi_{i}^{k}=1, \chi_{i}^{\dagger}=\chi_{i}^{k-1}$, and $\chi_{i} \chi_{j}=\exp (2 \pi i / k) \chi_{j} \chi_{i}$ algebra for $i<j[17,19]$. The $Z_{k}$ parafermion Read-Rezayi state can be obtained by condensing a cluster of $k$ parafermions. This condensation is allowed because the $k$ parafermion cluster behaves like a bosonic object. Fibonacci anyons emerge as the excitations above the condensate of $Z_{3}$ parafermions. For instance, they can bind to the topological defects of the condensate, e.g., inside vortices.

Recently, the problem of perturbing a counterpropagating edge mode of a fractional topological insulator [21] or two nearby FQH states with opposite spin polarizations by either electron pairing or backscattering has attracted a lot of attention. It has been shown that the parafermion (fractionalized Majorana fermion) zero modes can be obtained at the domain wall between regions with these different mass terms [6-8,22-27]. It has also been shown that parafermion zero modes can be found in the bulk of an FQH state that has acquired superconducting pairing through the proximity effect [9] as well as in other 2D systems [28-35].

After obtaining parafermions, a condensate of parafermions is needed to achieve a $Z_{k}$ parafermion state. In this paper, we show that this $Z_{k}$ parafermion superconductor can naturally happen in a fractional topological superconductor (FTSC), which is defined as an Abelian FQH state that has acquired superconducting pairing either intrinsically or through the proximity effect (see Fig. 1). The basic observation behind our idea is the way we obtain $Z_{k}$ parafermion $\mathrm{FQH}$ states. It is achieved by condensing clusters of $k$ quasiparticles. Now, imagine an Abelian FQH state at $\nu=2 / k$. It supports anyon excitations with $q_{a}=$ $2 e / k$ electric charge. We then induce pairing by some means into this state. The charge of the Cooper pair is $2 e$; therefore, we need $k$-anyon condensation. This condensate is topologically indistinguishable from the non-Abelian part of the Read-Rezayi $Z_{k}$ parafermion wave function.

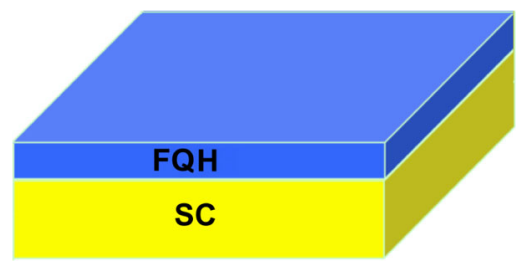

FIG. 1. Schematic setup of a fractional topological superconductor. This state of matter can be achieved by inducing a superconducting order parameter into a fractional quantum Hall bar or a fractional Chern insulator through the proximity effect.
The fractional Chern insulator (FCI) is a topological state of strongly interacting electrons residing in a partially filled nearly flatband with nontrivial Chern number [36-42]. The FCI has the same topological order as an $\mathrm{FQH}$, although there is no external magnetic field. Therefore, as long as the topological order of the parent state is concerned, we can substitute an FQH for an FCI without changing the final results. From now on, we do not distinguish between them, and one can replace an $\mathrm{FQH}$ with an FCI everywhere in this paper. It is worth mentioning that there are two huge advantages for the FCI compared to the $\mathrm{FQH}$ in our discussions. First, there is no external magnetic field that can kill superconductivity in an $\mathrm{FQH}$ system. Second, it is much easier to induce $s$-wave pairing in it.

These intriguing findings have motivated us to reexamine more carefully the superconductor (SC)-FQH heterostructure. However, the nature of this problem is difficult and highly nontrivial due to strong correlations in the FQH state. Nevertheless, we introduce a trick that can help us better understand this situation. We will discuss in Sec. II that a generic Abelian FQH state can be mapped into an array of one-dimensional (1D) chains of counterpropagating free bosons. These chains are coupled by anyon backscattering terms such that the bulk becomes fully gapped while boundaries host chiral gapless modes. A similar construction has been introduced by Teo and Kane to obtain $\mathrm{FQH}$ states by starting from decoupled Luttinger liquids. This type of mapping helps us to study the effect of superconducting pairing on these 1D chains more efficiently by utilizing powerful techniques of the CFT approach. CFT predicts that when $\left(\nu_{c} \bmod 1\right)<1 / 2$, the $1 \mathrm{D}$ theory of each chain flows to $Z_{k}$ parafermions in the infrared (IR) fixed point $[43,44]$, where $k=2 / \nu$. Interchain anyon backscattering gaps out the bulk of the system but maintains a chiral edge state described by $Z_{k}$ parafermion CFT around the sample.

It is worth mentioning that the $\mathrm{FQH}$ state has a nonzero bulk gap that prevents quantum phase transition as long as it is open. When the superconducting pairing is weak, the bulk gap does not close and no phase transition is expected. As the pairing becomes stronger and comparable to the many-body gap, we reach a quantum critical point. The system undergoes a topological phase transition into a different topological state at this point, and when the gap reopens, the nature of the state becomes completely different than its parent state. This type of problem was first studied by us in Ref. [9]. We showed there that the highenergy description of the FTSC can be studied by modifying the edge theory of the Abelian parent state. Assuming the parent state's edge theory is a $U(1)_{k} \mathrm{CFT}$, after the phase transition, it would be a $U(1)_{k} / Z_{2}$ orbifold CFT $[9,11,45-47]$. In this paper, we reinvestigate this problem and show that for $k<4$, the system has a different lowenergy description than its high-energy limit. For these 
values of $k$, we can find the superconducting analogue of the Read-Rezayi $Z_{k}$ parafermion state.

Our paper is organized as follows. In Sec. II, we briefly introduce the 2D bosonization of the Abelian FQH states by mapping them onto an array of $1 \mathrm{D}$ chains through applying a similar construction to that of Teo and Kane [48]. This mapping allows us to use powerful techniques of the CFT approach. Using the bosonization framework, we study the FQH-SC heterojunction and map this problem onto coupled 1D chains as well. In Sec. III, we focus on the Hamiltonian that describes a single chain. We discuss that the self-dual sine-Gordon (SDSG) model emerges as the effective Hamiltonian of the decoupled $1 \mathrm{D}$ chains. We show that by tuning the strength of interchain pairing and backscattering, we can achieve the self-dual point of the SDSG model, which is a quantum critical point. It is believed that this critical point is described by a $Z_{k}$ parafermion CFT for $k<4$. One mechanism that can enhance backscattering is to induce in-plane magnetic order in an FCI with strong spin-orbit coupling, which can be achieved by putting a ferromagnet on top of an FCI. In Sec. IV, we study the effect of interchain coupling and show that it gaps out every two nearby counterpropagating modes. These observations solve our main problem of finding the superconducting analogue of the Read-Rezayi $Z_{k}$ parafermion $\mathrm{FQH}$ state.

\section{BOSONIZATION OF ABELIAN FQH STATES AND FTSCs}

As we mentioned earlier, in the current paper, an FCI is smoothly connected to the $\mathrm{FQH}$ state in the presence of an external magnetic field. Therefore, they are described by the same topological order, i.e., their anyon excitations, edge states, and every other topological characteristic; e.g., the ground-state degeneracy is identical. In this section, the $\mathrm{FQH}$ at filling $\nu_{c}$ denotes all the gapped physical systems that can be smoothly transformed into one another, including the FCI.

The FQH effect requires the interaction between electrons to be much larger than their kinetic energies. Therefore, the FQH state is, by definition, a strongly correlated system, and any description of the system is approximate and far from being exact. Thus, the problem of the FQH-SC heterostructure is very challenging. In this section, we pause and try to overcome this difficulty first. We introduce a mapping that will turn out to be very useful in solving our problem. We demonstrate that an Abelian $\mathrm{FQH}$ state can be mapped into an array of coupled 1D chains of counterpropagating gapless modes, each described by a free-boson CFT. This mapping to coupled 1D chains allows us to take advantage of the powerful techniques of the CFT approach in solving our main problem.

The basic observation behind our scheme is that the interior of an Abelian $\mathrm{FQH}$ state is gapped while its (a)

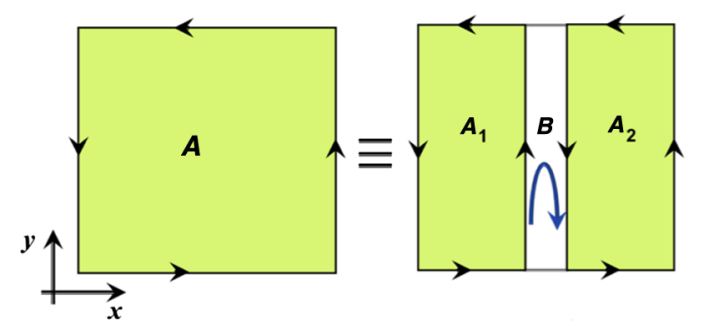

(b)

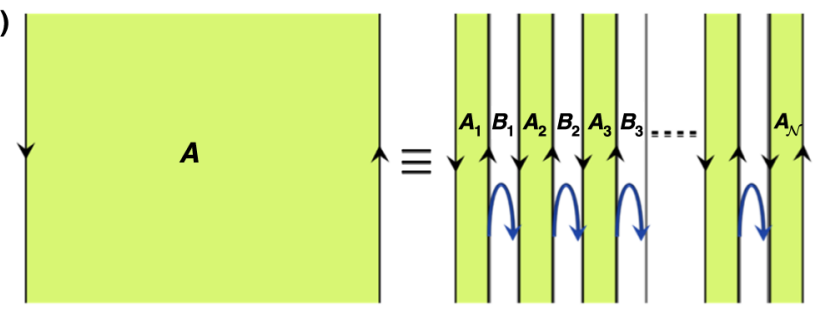

FIG. 2. An Abelian FQH state can be imagined as an array of coupled one-dimensional chains, each described by a free-boson CFT. (a) FQH region $A$ can be split into regions $A_{1}$ and $A_{2}$ in addition to the extremely narrow vacuum region $B$. The bent blue arrow represents the backscattering process between the two counterpropagating gapless modes. (b) By repeating this procedure, we can map a 2D FQH state into an array of narrow strips $A_{1}$ to $A_{\mathcal{N}}$ that are coupled to one another through backscattering. Each narrow strip can be imagined as a 1D chain with a freeboson description.

boundary with a trivial insulator, e.g., vacuum, supports gapless edge modes described by a free-boson CFT [49]. Furthermore, we assume that every two systems with gapped bulk and the same edge theory are described by the same topological order. Next, we cut the FQH system into three pieces: $A_{1}$ and $A_{2}$, which are separated by a third trivial narrow region $B$ (see Fig. 2) whose length along the $x$ axis will be set to 0 at the end of the process. Since both the $A_{1}$ and $A_{2}$ regions are surrounded by vacuum, each supports a gapless chiral edge mode. Therefore, there are two counterpropagating modes on the two sides of region $B$. It should be emphasized that these two counterpropagating modes can have identical center-of-mass momenta. Because we take the narrow limit of the intervening region, the two counterpropagating modes can couple to one another through backscattering. It is well known that the backscattering between two identical counterpropagating modes can gap them out [50,51]. Therefore, there is no gapless edge state on the two sides of region $B$ after taking the backscattering process into consideration. This result is consistent with our intuition of an FQH state that can have a gapless edge state only at its boundary with a different topological state.

The above procedure can be easily generalized by splitting the system into $\mathcal{N} \mathrm{FQH}$ regions $A_{I}$ that are separated by narrow vacuum regions $B_{I}$ (see Fig. 2). After taking the $\mathcal{N} \rightarrow \infty$ limit, the narrow $\mathrm{FQH}$ liquid region $A_{I}$ becomes a $1 \mathrm{D}$ chain with two counterpropagating modes. These chains are coupled by backscattering through 
$B_{I}$ regions. This simple trick motivates us to imagine an $\mathrm{FQH}$ state as an array of coupled 1D chains, thus suggesting a CFT description of the system. To summarize, we first introduce an infinite number of narrow strips, each with two counterpropagating modes. The strips acquire mass through backscattering between adjacent counterpropagating modes. In this way, the bulk of the system becomes gapped. If the two outermost modes at the boundary of the original sample remain gapless, the system corresponds to an $\mathrm{FQH}$; otherwise, it represents a trivial insulator with trivial topological order. In the rest of this paper, we demonstrate how powerful this technique is in understanding the superconducting proximity effect on the Abelian FQH states.

\section{A. Bosonization of fractional quantum states}

Consider an Abelian $\mathrm{FQH}$ state of electrons at filling fraction $\left|\nu_{c}\right|=2 / k$ where $N \in \mathbb{Z}$. For $k=2 m$, it can be a monolayer, while for $k=2 m+1$, we consider a bilayer system, e.g., $(k, k, 0),(k-1, k-1,1)$, or $(1,1, k-1)$ Halperin states. The multicomponent quantum Hall states can have extended symmetries. Hence, excitations carry different charges, for example, pseudospin (associated with layer index), spin, and electric charge. From now on, for two reasons, we focus on the charged sector of the system and charged degrees of freedom only. First, neutral fluctuations are unaffected by inducing superconducting pairing and do not modify our results. Second, we need to somehow gap out the neutral fluctuations for a simple reason. The minimum charge of excitations in the Halperin states mentioned earlier is $\nu_{c} / 2=1 / k$ rather than $\nu_{c}=2 / k$. However, we need $k$-anyon condensation in order to obtain a $Z_{k}$-symmetric state; hence, the minimum charge of excitation should become twice as large. This behavior can be achieved through binding anyons of the top and bottom layers, i.e., gapping the pseudospin excitation.

Before discussing the bosonization procedure, let us pause and briefly describe how we can decouple charge and neutral degrees of freedom in the $(n n l)$ two-component quantum Hall state with a $\nu_{c}=2 / n+l$ filling fraction. Using the $K$-matrix formulation, there are $\operatorname{det}(K)=n^{2}-l^{2}$ nontrivial anyon excitations described by integer-valued $\vec{p} \equiv\left(p_{1}, p_{2}\right) \quad$ vectors subject to $\vec{p} \sim \vec{p}+(n, l)$ and $\vec{p} \sim \vec{p}+(l, n)$ identifications. The self-statistics of $\vec{p}$ excitation is $\theta_{p, p}=\pi \mathbf{p}^{T} \mathbf{K}^{-1} \mathbf{p}$, and the mutual statistics between $\vec{p}$ and $\vec{q}$ distinct excitations is $\theta_{\mathbf{p}, \mathbf{q}}=2 \pi \mathbf{p}^{T} \mathbf{K}^{-1} \mathbf{q}$ [49]. There are two electron operators given by $(n, l)$ and $(l, n)$ vectors. In the edge CFT picture, the electron operators can be bosonized as $c_{+}=e^{i\left(n \phi_{1}+l \phi_{2}\right)}$ and $c_{-}=e^{i\left(l \phi_{1}+n \phi_{2}\right)}$ with the $\left\langle\phi_{i}(z) \phi_{j}(w)\right\rangle=\left(K^{-1}\right)_{i j} \log (z-w)$ correlation function. For the $(n n l)$ state, we can define the charged and neutral chiral bosons as $\phi_{c}=\left(\phi_{1}+\phi_{2}\right)$ and $\phi_{s}=\left(\phi_{1}-\phi_{2}\right)$. Thus, the two electron operators are represented by $c_{+}=$ $e^{i\left(\phi_{c} / \nu_{c}+\phi_{s} \nu_{s}\right)}$ and $c_{-}=e^{i\left(\phi_{c} / \nu_{c}-\phi_{s} / \nu_{s}\right)}$ in terms of charged and neutral degrees of freedom where $\nu_{c}=2 / n+l$ and $\nu_{s}=2 / n-l$. Later, we imagine a situation where the neutral sector is gapped. This assumption allows us to drop the neutral boson from expressions, and both electron operators can be represented as $c \propto e^{i \phi_{c} / \nu_{c}}$, where $\nu_{c}$ is the filling fraction.

Now, let us come back to our coupled-wire construction of the $\mathrm{FQH}$ states. The procedure explained in the previous section suggests the following description for the "charged sector" of an Abelian FQH state at filling fraction $\nu_{c}$ in terms of coupled $1 \mathrm{D}$ chains $[7,52]$

$$
\begin{aligned}
H_{0} & =\int d x\left(\mathcal{H}_{0}+\mathcal{H}_{1}^{\mathrm{BS}}+\mathcal{H}_{2}^{\mathrm{BS}}\right), \\
\mathcal{H}_{0} & =\sum_{I=1}^{\mathcal{N}} \sum_{\tau=c, s} \frac{\nu_{\tau}^{-1}}{4 \pi}\left[\left(\partial_{x} \phi_{\tau R}^{I}\right)^{2}+\left(\partial_{x} \phi_{\tau L}^{I}\right)^{2}\right] \\
\mathcal{H}_{1}^{\mathrm{BS}} & =-\sum_{I=1}^{\mathcal{N}} g_{I, I}^{\mathrm{BS}} \cos \left(\frac{\phi_{c R}^{I}-\phi_{c L}^{I}}{\nu_{c}}\right) \cos \left(\frac{\phi_{s R}^{I}-\phi_{s L}^{I}}{\nu_{s}}\right), \\
\mathcal{H}_{2}^{\mathrm{BS}} & =-\sum_{I=1}^{\mathcal{N}-1} g_{I, I+1}^{\mathrm{BS}} \cos \left(\frac{\phi_{c R}^{I}-\phi_{c L}^{I+1}}{\nu_{c}}\right) \cos \left(\frac{\phi_{s R}^{I}-\phi_{s L}^{I+1}}{\nu_{s}}\right),
\end{aligned}
$$

where $\exp \left(i \phi_{R(L)}^{c I} / \nu_{c} \pm \phi_{R(L)}^{s I} / \nu_{s}\right)$ denotes the bosonized right-moving (left-moving) electron operator on the upper (lower) layer and the $I$ th chain. Here, $\mathcal{H}_{1}^{\mathrm{BS}}$ represents the intrachain backscattering of electrons and $\mathcal{H}_{2}^{\mathrm{BS}}$ interchain electron backscattering. In principle, the "intrachain anyon backscattering" process is allowed and must be taken into account in our analysis. However, as we will discuss shortly, for the setup we study in this paper, they are subdominant terms and we will neglect them in our discussion. On the other hand, the interchain anyon backscattering is strictly disallowed since anyons cannot pass through the vacuum (see Fig. 2).

In the quantum Hall regime, $\mathcal{H}_{1}^{\mathrm{BS}}$ as well as intrachain anyon backscattering terms should be either absent or weak enough; otherwise, all chains become gapped and there will be no edge state. However, there are ways to generate intrachain electron backscattering $\left(\mathcal{H}_{1}^{\mathrm{BS}}\right)$. For example, inducing an in-plane magnetic order in the fractional Chern insulator with strong spin-orbit coupling through proximity to a ferromagnet can enhance electron backscattering. The presence of strong spin-orbit coupling leads to (partial) spin-momentum locking; hence, spin flip will amount to backscattering of electrons. This effect does not enhance anyon backscattering. The reason is that anyon backscattering through the proximity effect requires anyon excitation in the substrate as well, whereas a ferromagnet is a topologically trivial state and does not support anyon excitations. Therefore, in this paper, we make the central assumption that proximity-induced intrachain electron backscattering is much stronger than intrinsic intrachain 
anyon backscattering because the proximity effect can enhance electron backscattering only. The above simple model in Eq. (1) can describe the phase transition between the $\mathrm{FQH}$ and the trivial state. For instance, when $g_{I, I} \ll g_{I, I+1}$, we can ignore $\mathcal{H}_{1}^{\mathrm{BS}}$, so $\phi_{L}^{1}$ and $\phi_{R}^{N}$, which live at the boundaries of the system, remain gapless, while all other modes become massive. Therefore, we obtain an FQH state for $g_{I, I} \ll g_{I, I+1}$. In the opposite limit, where $g_{I, I} \gg g_{I, I+1}$, we can ignore $\mathcal{H}_{2}^{\mathrm{BS}}$, and due to strong intrachain backscattering, the system is completely gapped, resulting in a trivial insulator.

\section{B. Bosonization of fractional topological superconductors}

We now wish to study the effect of superconductivity on an Abelian FCI state at filling fraction $\nu_{c}=2 / k$. To this end, we assume the spin-orbit coupling is strong in the system, and thus the electron spin is correlated with its momentum. In other words, the spin of the $c_{ \pm, R}^{I} \sim$ $e^{i \phi_{c R}^{I} / \nu_{c} \pm i \phi_{s R}^{I} / \nu_{s}}$ electron operator is opposite to that of $c_{ \pm, L}^{I} \sim e^{i \phi_{L}^{I} / \nu_{c} \pm i \phi_{s L}^{I} / \nu_{s}}$. Consequently, they can be paired through $s$-wave pairing. This $s$-wave superconductivity can be either intrinsic or induced through the proximity effect. We consider two perturbations: intrachain and interchain pairings that have $g_{I, I}^{\mathrm{SC}} c_{R}^{I} c_{L}^{I}$ and $g_{I, I+1}^{\mathrm{SC}} c_{R}^{I} c_{L}^{I+1}$ structures, respectively. These terms have the following bosonized representations:

$$
\begin{aligned}
\mathcal{H}_{1}^{\mathrm{SC}} & =-\sum_{I=1}^{\mathcal{N}} g_{I, I}^{\mathrm{SC}} \cos \left(\frac{\phi_{c R}^{I}+\phi_{c L}^{I}}{\nu_{c}}\right) \cos \left(\frac{\phi_{s R}^{I}-\phi_{s L}^{I}}{\nu_{s}}\right), \\
\mathcal{H}_{2}^{\mathrm{SC}} & =-\sum_{I=1}^{\mathcal{N}-1} g_{I, I+1}^{\mathrm{SC}} \cos \left(\frac{\phi_{c R}^{I}+\phi_{c L}^{I+1}}{\nu_{c}}\right) \cos \left(\frac{\phi_{s R}^{I}-\phi_{s L}^{I+1}}{\nu_{s}}\right) .
\end{aligned}
$$

Therefore, the Hamiltonian density of an FTSC is $\mathcal{H}_{0}+\mathcal{H}_{1}^{\mathrm{BS}}+\mathcal{H}_{2}^{\mathrm{BS}}+\mathcal{H}_{1}^{\mathrm{SC}}+\mathcal{H}_{2}^{\mathrm{SC}}$. Now, let us define the following bosonic variables:

$\varphi_{c / s}^{I}=\frac{\phi_{c / s, R}^{I}+\phi_{c / s, L}^{I}}{2}, \quad \theta_{c / s}^{I}=\frac{\phi_{c / s, R}^{I}-\phi_{c / s, L}^{I}}{2}$,

with

$$
\begin{aligned}
& {\left[\varphi_{\tau}^{I}(x, t), \varphi_{\tau^{\prime}}^{I}(y, t)\right]=0, \quad\left[\theta_{\tau}^{I}(x, t), \theta_{\tau^{\prime}}^{I}(y, t)\right]=0,} \\
& {\left[\varphi_{\tau}^{I}(x, t), \theta_{\tau^{\prime}}^{I}(y, t)\right]=i \frac{\nu_{\tau} \pi}{2} \delta_{\tau, \tau^{\prime}} \operatorname{sgn}(x-y)}
\end{aligned}
$$

equal-time commutation relations. On one hand, the above bosonic variables tend to fluctuate due to the kinetic term $\mathcal{H}_{0}$. On the other hand, in order to gain potential energy, the argument of the cosine perturbations must freeze, i.e., be replaced by a constant value ( $c$ number) at every spatial point. If the latter happens, then the sector associated with that bosonic variable becomes massive. However, this wish cannot always be fulfilled. For example, if we condense the $\varphi_{c}^{I}(x, t)$ boson (i.e., replace it by its expectation value) such that $\left|\cos \left(2 \varphi_{c}^{I} / \nu_{c}\right)\right|=1$, then we cannot have $\left|\cos \left(2 \theta_{c}^{I} / \nu_{c}\right)\right|=1$ because $\left[\varphi_{c}^{I}(x, t), \theta_{c}^{I}(y, t)\right] \neq 0$, and these two conjugate variables cannot simultaneously condense. An important observation about the above Hamiltonians is that they contains $\cos \left(2 \theta_{s}^{I} / \nu_{s}\right)$ only, and, as a result, we can maximize it by assuming $\theta_{s}^{I}=n \nu_{s} \pi$, where $n$ is an integer number. Thus, the neutral sector is gapped out. For this reason, from now on, we focus on the charged sector and investigate the effect of having two incompatible perturbations $\cos \left(2 \varphi_{c}^{I} / \nu_{c}\right)$ and $\cos \left(2 \theta_{c}^{I} / \nu_{c}\right)$.

In the absence of interchain interaction and after condensing $\theta_{s}^{I}$ neutral bosons, the above Hamiltonian reduces to an array of $\mathcal{N} \rightarrow \infty$ decoupled 1D chains, each described by a SDSG Hamiltonian [43]:

$$
\begin{aligned}
\mathcal{H}_{\mathrm{SG}}^{I}= & \frac{\nu_{c}^{-1}}{2 \pi} \int d x\left[\left(\partial_{x} \varphi_{c}^{I}\right)^{2}+\left(\partial_{x} \theta_{c}^{I}\right)^{2}\right], \\
& -\int d x\left[g_{I, I}^{* \mathrm{SC}} \cos \left(2 \varphi_{c} / \nu_{c}\right)+g_{I, I}^{* \mathrm{BS}} \cos \left(2 \theta_{c} / \nu_{c}\right)\right],
\end{aligned}
$$

where $g_{I, I}^{* \mathrm{SC}(\mathrm{BS})}=g_{I, I}^{\mathrm{SC}(\mathrm{BS})}\left\langle\cos \left(2 \theta_{s}^{I} / \nu_{s}\right)\right\rangle$. In the following section, we first try to review the properties of the above well-studied SDSG model $[43,44]$. We then investigate the effect of coupling between different 1D chains that are described by the above SDSG Hamiltonian. This scheme will help us to find a route for finding the superconducting analogue of the Read-Rezayi parafermion wave function.

\section{PHASE DIAGRAM OF DECOUPLED 1D CHAINS}

In this section, we focus on one chain of two counterpropagating modes perturbed by superconducting pairing and electron backscattering. As we mentioned in the previous section, the resulting Hamiltonian has the SDSG form. So, let us consider the following SDSG Hamiltonian of a 1D system:

$$
\begin{aligned}
\mathcal{H}_{\mathrm{SG}}(k)= & \frac{k}{4 \pi} \int d x\left[\left(\partial_{x} \varphi\right)^{2}+\left(\partial_{x} \theta\right)^{2}\right], \\
& -\int d x\left[g_{1} \cos (k \varphi)+g_{2} \cos (k \theta)\right] .
\end{aligned}
$$

By comparing the above equation with Eq. (5), we see that $k$ is related to the filling fraction of the parent $\mathrm{FQH}$ state as follows: $k=2 / \nu_{c}$. The first part of the SDSG Hamiltonian describes a free-boson theory with $c=1$ compactified on a circle with radius $R=\sqrt{k / 2}$. The second part of the SDSG Hamiltonian contains two different types of mass terms that do not commute with each other, since 


$$
\left[\varphi(x), \theta\left(x^{\prime}\right)\right]=i \frac{\pi}{k} \operatorname{sgn}\left(x-x^{\prime}\right) .
$$

The conformal dimension of the $g_{1}$ as well as the $g_{2}$ term is $k / 2$. Therefore, they are relevant perturbations when $k<4$. The cosine perturbations break the $U(1) \times U(1)$ symmetry associated with the kinetic part of the SDSG model to its $Z_{k} \times Z_{k}^{\text {dual }}$ subgroup generated by

$$
Z_{k}: \varphi \rightarrow \varphi+2 \pi / k, \quad \theta \rightarrow \theta,
$$

and

$$
Z_{k}^{\text {dual }}: \theta \rightarrow \theta+2 \pi / k, \quad \varphi \rightarrow \varphi,
$$

respectively. Interestingly, the $Z_{k}$ clock model also enjoys two distinct $Z_{k}$ symmetries. In fact, it can be shown that the SDSG model with index $k$ describes the continuum limit of the $Z_{k}$ clock model [53].

Some useful insights can be obtained by studying the SDSG model at its limiting cases. For instance, for $g_{2}=0$, the charged $\varphi$ condenses at one of its classical values, i.e., $\varphi_{0}=2 \pi m / k$. This condensation can happen since $\left[\varphi(x), \varphi\left(x^{\prime}\right)\right]=0$. Similarly, when $g_{1}=0$, the $\theta$ field condenses to its classical values $\theta_{0}=2 \pi m / k$ because $\left[\theta(x), \theta\left(x^{\prime}\right)\right]=0$. Let us define the following order parameters:

$$
\begin{aligned}
& \sigma(z, \bar{z}) \sim e^{i \varphi(z, \bar{z})}+\alpha e^{-i(k-1) \varphi(z, \bar{z})}, \\
& \mu(z, \bar{z}) \sim e^{i \theta(z, \bar{z})}+\beta e^{-i(k-1) \theta(z, \bar{z})},
\end{aligned}
$$

where $\alpha$ and $\beta$ are two nonuniversal constants. The $\sigma$ order parameter (also known as the spin field) carries unit charge of the first $Z_{k}$ and is neutral under the dual $Z_{k}$ symmetry, while the $\mu$ order parameter (also known as the spin disorder field) is neutral under $Z_{k}$ and carries unit charge of the $Z_{k}^{\text {dual }}$. These two order parameters do not commute and cannot condense simultaneously. Therefore, we can distinguish two gapped phases by looking at the expectation values of the $\sigma$ and $\mu$ fields. The so-called ferromagnetic phase $\left(g_{1} \gg g_{2}\right)$ is characterized by $\langle\sigma\rangle \neq 0$ and $\langle\mu\rangle=0$, while in the paramagnetic phase, $\left(g_{1} \ll g_{2}\right)$ is characterized by $\langle\mu\rangle \neq 0$ and $\langle\sigma\rangle=0$.

The SDSG model at $g_{1} g_{2}=0$ reduces to the $X Y$ model of a system with in-plane magnetic order that has a single global $U(1)$ symmetry associated with either the $\varphi$ or $\theta$ field and a discrete $Z_{k}$ symmetry associated with the other one. On the other hand, the $g_{2}\left(g_{1}\right)$ mass term can be imagined as the vortex operator for the $g_{1}\left(g_{2}\right)$ mass term. This vortex operator breaks the mentioned $U(1)$ symmetry down to its $Z_{k}$ subgroup. To see this symmetry breaking, we need to consider the following commutation relation:

$$
\left[\varphi(x), e^{-i k \theta\left(x^{\prime}\right)}\right]=\pi \operatorname{sgn}\left(x-x^{\prime}\right) e^{-i k \theta\left(x^{\prime}\right)} .
$$

As a result, if we label a ground state by $\varphi(x)\left|\varphi_{0}\right\rangle=$ $\varphi_{0}\left|\varphi_{0}\right\rangle$, we have

$$
\begin{array}{rlrl}
e^{-i k \theta\left(x^{\prime}\right)} \mid \varphi_{0} & >\sim\left|\varphi_{0}+\pi\right\rangle, & & x^{\prime}<x, \\
\left.e^{-i k \theta\left(x^{\prime}\right)}\left|\varphi_{0}>\sim\right| \varphi_{0}-\pi\right\rangle, & & x^{\prime}>x,
\end{array}
$$

which simply means that $e^{-i k \theta\left(x^{\prime}\right)}$ creates a kink in the profile of $\varphi_{0}$ at $x^{\prime}$ and shifts its value by $2 \pi$ at that point. Consequently, the $g_{2}$ term creates vortices and compactifies the $\varphi$ field as

$$
\varphi \sim \varphi+2 \pi
$$

Because of this periodicity, $\varphi_{0}$ has only $k$ distinct values given by

$$
\varphi_{0}=\frac{2 \pi m}{k}, \quad m=0, \ldots, k-1 .
$$

Therefore, when $g_{1} \gg g_{2}, \varphi$ condenses and the freeboson theory becomes massive. Similarly, when $g_{2} \gg g_{1}, \theta$ condenses and we again obtain a gapped theory. However, the situation is fundamentally different at the $\left|g_{1}\right|=\left|g_{2}\right|$ point. In this case, the theory is symmetric under the $\varphi \leftrightarrow \theta$ exchange and shows self-duality. At this point, neither $\varphi$ nor $\theta$ can condense or is a critical point $[43,44,48]$.

At the ultraviolet (UV) fixed point, the SDSG model is a CFT with $c_{\mathrm{UV}}=1$. At the IR limit, the SDSG model is critical at the self-dual point and described by a CFT. On the other hand, according to the $c$ theorem [54], when we perturb a CFT with relevant operators [in the sense of renormalization group (RG)], i.e., when $k<4$ in our problem (so the scaling dimension of the mass terms does not exceed 2), then $c_{\mathrm{IR}}<c_{\mathrm{UV}}=1$. Hence, the IR fixed point is necessarily described by a minimal CFT. At this IR fixed point, the $g_{1}$ and $g_{2}$ terms compete with each other and do not let the other term condense. This behavior can be seen through the vortex picture. We can approach the self-dual point from the $g_{1}>g_{2}$ side. Along that direction, the $g_{1}$ term dominates over the $g_{2}$ term, and thus $\cos (k \varphi)$ maximizes through picking one of the $k$ distinct values of $\varphi$. Since $\theta$ and $\varphi$ are conjugate fields, the $g_{2} \cos (k \theta)$ term creates vortices for the classical configuration of $\varphi_{0}(x)$. Therefore, $g_{2}$ can be considered as the fugacity of the vortex gas. When $g_{2}$ approaches $g_{1}$, vortices proliferate all over the system and $\varphi$ fluctuates strongly. Thus, at $g_{2}=g_{1}$, a second-order phase transition of the BerezinskyKosterlitz-Thouless (BKT) type (vortex proliferation) is expected. Since $\varphi_{0}$ can take $k$ distinct values, the universality class of the phase transition is identical to that of the $Z_{k}$ clock model or equivalently to that of a $k$-state Potts model. The critical point of these theories is described by a $Z_{k}$ parafermion CFT [43]. Therefore, we expect the SDSG model to be described by a $Z_{k}$ parafermion CFT at its IR fixed point for $k<4$. 
When $k>4$, both the $g_{1}$ and $g_{2}$ terms are irrelevant; therefore, $c_{\mathrm{UV}}=c_{\mathrm{IR}}$. It is known that for $u<u_{c 1}<1$, where $u=g_{2} / g_{1}, \varphi$ condenses, while for $u>u_{c 2}>1$, the conjugate field $\theta$ is condensed. For these values of $k$, instead of having a single critical point, the system is gapless with Gaussian fluctuations all over the so-called Villain line: $u_{c 1}<u<u_{c 2}[55,56]$. Furthermore, Fendley showed that after a generalized Jordan-Wigner transformation, the $Z_{k}$ chiral quantum clock model transforms to a dual theory of a $Z_{k}$ parafermion chain that exhibits an end-state $d=\sqrt{k}$ quantum dimension when the original clock model is in its ordered phase [19]. So, we conclude that the CFT description of the SDSG must allow for a primary field with the same quantum dimension.

For $k<4$, the UV fixed point of the SDSG model, i.e., the unperturbed Hamiltonian (in which $g_{1}=g_{2}=0$ ), is described by the $U(1)_{k}$ free-boson CFT whose central charge is $c=1$. Since the perturbations in the SDSG model are relevant for $k<4$, the IR fixed point of the SDSG model has a different CFT description. For example, for $k=2$, we achieve a single Ising CFT ( $Z_{2}$ parafermion) and a $Z_{3}$ parafermion CFT for $k=3$ in the IR limit. Furthermore, noting the facts that the $U(1)_{k} / Z_{2}$ orbifold CFT has $Z_{k}$ symmetry, $c=1$ central charge, and twist fields with quantum dimension $d_{\mathrm{tw}}=\sqrt{k}$ that trap the $Z_{k}$ parafermion zero mode [9], we conjecture that the gapless region of the SDSG for $k>4$ is described by the $U(1)_{k} / Z_{2}$ orbifold theory.

Before closing this section, it is worth mentioning that the "parafermion primary field" $\psi_{1, R}$ of the $Z_{k}$ parafermion CFT carries unit charge of both $Z_{k}$ and $Z_{k}^{\text {dual }}$ symmetries in Eq. (8). Similarly, the $\psi_{1, L}$ carries $+1 Z_{k}$ charge and $-1 Z_{k}^{\text {dual }}$ charge [53]. This observation suggests the following identifications in terms of the UV fixed-point freeboson fields:

$\psi_{1, R} \sim \sigma(z, \bar{z}) \mu(z, \bar{z}) \sim e^{i \phi_{R}}+\alpha^{\prime} e^{-i(k-1) \phi_{R}}+\cdots$,

$\psi_{1, L} \sim \sigma(z, \bar{z}) \mu^{\dagger}(z, \bar{z}) \sim e^{i \phi_{L}}+\alpha^{\prime} e^{-i(k-1) \phi_{L}}+\cdots$,

where the centered dots denote higher-order (less relevant) terms. The above relations must be viewed as the "transmutation" of the UV primary fields with a free-boson description to the primary fields of the IR fixed point under RG flow. In other words, if we start from the vertex operator on the right side in the UV limit, then the RG flow will transform it into the left side as we approach the IR fixed point. We must stress that the lattice parafermion operator that is the building block of some statistical models is different from the parafermion primary field, and these two should not be confused. The lattice parafermion does not have a definite chirality, is not a primary field, and has a more complicated UV-IR transmutation. (See Ref. [53] for more details.)

For some specific values of $k$, the SDSG model can be solved exactly $[43,48]$. In the following, we will first describe the exactly solvable $k=2$ SDSG and then discuss the more interesting $k=3$ case.

\section{A. $\boldsymbol{k}=\mathbf{2}$ self-dual sine-Gordon model}

At $k=2$, it is possible to fermionize the SDSG model. This fermionization can be seen from the scaling dimension of the $\cos (2 \theta)$ and $\cos (2 \varphi)$ operators, which is unity. These mass terms can be written as a fermion bilinear since the scaling dimension of the holomorphic part of the free electrons is $1 / 2$. So, we are dealing with a noninteracting problem that can be easily solved $[43,57]$. To this end, we define the following fermionic operators:

$c_{R} \propto e^{i \phi_{R}}=e^{i(\varphi+\theta)}, \quad c_{L} \propto e^{i \phi_{L}}=e^{i(\varphi-\theta)}, \quad k=2$.

Using the above definitions, we can rewrite $\mathcal{H}_{1}$ as

$$
\begin{aligned}
\mathcal{H}_{1}= & -1 / 2 \int d x g_{1}\left(e^{i\left(\phi_{R}+\phi_{L}\right)}+e^{-i\left(\phi_{R}+\phi_{L}\right)}\right) \\
& +g_{2}\left(e^{i\left(\phi_{R}-\phi_{L}\right)}+e^{-i\left(\phi_{R}-\phi_{L}\right)}\right) .
\end{aligned}
$$

It is convenient to work with Majorana fermions defined as follows:

$$
\begin{aligned}
& \gamma_{1, R(L)}=\frac{c_{R(L)}+c_{R(L)}^{\dagger}}{2}=\cos \left(\phi_{R(L)}\right), \\
& \gamma_{2, R(L)}=\frac{c_{R(L)}-c_{R(L)}^{\dagger}}{2 i}=\sin \left(\phi_{R(L)}\right) .
\end{aligned}
$$

In the Majorana-fermion basis, the sine-Gordon equation for $N=2$ is

$$
\begin{aligned}
\mathcal{H}_{\mathrm{SG}}^{N=2} & =\mathcal{H}_{0}+\mathcal{H}_{1}, \\
\mathcal{H}_{0} & =i \int d x \sum_{i=1,2}\left(\gamma_{i, R} \partial_{x} \gamma_{i, R}-\gamma_{i, L} \partial_{x} \gamma_{i, L}\right), \\
\mathcal{H}_{1} & =i \int d x\left[\left(g_{1}+g_{2}\right) \gamma_{1, R} \gamma_{1, L}-\left(g_{1}-g_{2}\right) \gamma_{2, R} \gamma_{2, L}\right],
\end{aligned}
$$

where $i$ in the $\mathcal{H}_{1}$ can be derived by carefully taking the zero mode of the Majorana fermions [48]. At the self-dual point, the interaction Hamiltonian creates the $2 i g_{1} \gamma_{1, R} \gamma_{1, L}$ mass term for the $\gamma_{1, R}$ and $\gamma_{1, L}$ Majorana fermions and leaves the other two Majorana fermions gapless. This result is consistent with our expectation for the $k=2$ SDSG to be described by a $Z_{2}$ parafermion theory, which happens to be the Ising CFT with Majorana fermions as its primary operators.

Now, consider an array of coupled 1D chains at the $g_{1}=g_{2}$ self-dual point. It is clear from Eq. (19) that the $\gamma_{1, R(L)}$ Majorana fermion is gapped, while $\gamma_{2, R(L)} \sim$ $\sin \left(\phi_{R(L)}\right)$ remains gapless. We then turn on the coupling 
between neighboring $\gamma_{2, R(L)}$ Majorana fermions. The generic term is of the $i g_{I, I+1} \gamma_{2, R}^{I} \gamma_{2, L}^{I+1}$ form, where $I$ stands for the index of the chain. This term gaps out all the Majorana fermions except for the two outermost Majorana modes, i.e., $\gamma_{2, L}^{1}$ and $\gamma_{2, R}^{N}$. The bosonized version of interchain coupling is as follows:

$$
\begin{aligned}
2 i \gamma_{2, R}^{I} \gamma_{2, L}^{I+1} & =2 \sin \left(\phi_{R}^{I}\right) \sin \left(\phi_{L}^{I+1}\right) \\
& =\cos \left(\phi_{R}^{I}+\phi_{L}^{I+1}\right)-\cos \left(\phi_{R}^{I}-\phi_{L}^{I+1}\right) .
\end{aligned}
$$

It is worth mentioning that the $i \gamma_{1, R}^{I} \gamma_{1, L}^{I+1}=$ $\cos \left(\phi_{R}^{I}\right) \cos \left(\phi_{L}^{I+1}\right)$ term can be added to the above mass term without causing a phase transition. As we mentioned above, $k=2 / \nu_{c}$; if we start from the $\nu_{c}=1$ quantum Hall state, we obtain $k=2$. The above mapping tells us that inducing pairing in a $\nu_{c}=1$ integer quantum Hall state will give us a topological superconductor with Ising anyons. This result can also be shown by starting from a lattice model with $C=1$ Chern number and adding a pairing term. The model can be solved exactly, after which it can be shown that there is a range of parameters in which we obtain a topological superconductor with a single Majorana edge state $[58,59]$.

\section{B. $\boldsymbol{k}=\mathbf{3}$ self-dual sine-Gordon model}

The SDSG model at $k=3$ can be shown to flow toward a $Z_{3}$ parafermion CFT at its IR fixed point [43,44,60-62]. One way to show this claim is to start from a $Z_{4}$ parafermion theory that can be bosonized. Then, by adding an appropriate mass term of the form $: \cos (3 \theta):+$ : $\cos (3 \varphi)$ : it can be shown that the theory will flow to the $\mathcal{M}_{5}$ minimal model, which is a $Z_{2}$ orbifold of the $Z_{3}$ parafermion CFT [43]. Interestingly, the primary fields of the $Z_{4}$ parafermion theory transmute under this UV-IR flow as well. Therefore, we can use this UV-IR transmutation to represent the primary fields of the $Z_{3}$ parafermion in terms of the primaries of the $Z_{4}$ theory with free-boson representation. In Ref. [[53]], we obtain all the primary fields of the $Z_{3}$ parafermion theory in terms of current and vertex operators. For example, the following identifications will be shown:

$$
\begin{aligned}
\cos \left(3 \phi_{R} / 2\right) \cos \left(3 \phi_{L} / 2\right) & \sim X(z, \bar{z}), \\
\sin \left(3 \phi_{R} / 2\right) \sin \left(3 \phi_{L} / 2\right) & \sim \epsilon(z, \bar{z}),
\end{aligned}
$$

where $\phi_{R(L)}=\varphi \pm \theta$ are chiral bosons, $X(z, \bar{z})$ is the neutral operator, and $\epsilon(z, \bar{z})$ is the thermal (energy) operator of the $Z_{3}$ parafermion theory with $\left(h_{X}, \bar{h}_{X}\right)=(7 / 5,7 / 5)$ and $\left(h_{\epsilon}, \bar{h}_{\epsilon}\right)=(2 / 5,2 / 5)$ conformal dimensions, respectively [11]. Adding a neutral operator is an irrelevant perturbation for the resulting IR fixed-point CFT. The above relations are consistent with the facts that (1) both thermal and neutral primary fields are neutral under the $Z_{3}$ and $Z_{3}^{\text {dual }}$ symmetries introduced in Eq. (8).
(2) The thermal operator is odd under the duality transformation $\varphi \leftrightarrow \theta$, and the neutral operator is even. (3) The $\cos \left(3 \phi_{R} / 2\right) \cos \left(3 \phi_{L} / 2\right)$ perturbation drives the phase transition between $Z_{4}$ and $Z_{3}$ theory. (4) Adding the $\sin \left(3 \phi_{R} / 2\right) \sin \left(3 \phi_{L} / 2\right)$ term to the $N=3$ SDSG model spoils the self-duality condition and results in a gapped theory. The thermal operator has zero spin and can be written as the product of its holomorphic and antiholomorphic parts. The above relations suggest the following identifications:

$$
\sin \left(3 \phi_{R} / 2\right) \sim \epsilon_{R}, \quad \sin \left(3 \phi_{L} / 2\right) \sim \epsilon_{L}
$$

It is worth mentioning that the $\epsilon_{R}^{I}$ and $\epsilon_{L}^{I+1}$ operators are not local with respect to themselves since their conformal spins are fractional, which amounts to a branch cut in their selfcorrelation functions. However, $\epsilon_{R}^{I} \epsilon_{L}^{I+1}$ has zero conformal spin, and there is no branch cut in its correlation with itself. So, it generates a local perturbation. Recall that the chiral and antichiral electron operators have been bosonized as

$$
c_{ \pm, R(L)}=e^{i\left[\left(3 \phi_{c R(L)}\right) / 2\right]} e^{i \pm\left[\left(\phi_{s R(L)}\right) / \nu_{s}\right]}, \quad k=3,
$$

in the UV limit and at $\nu_{c}=2 / 3$ filling. (Recall that in our notation, $c_{+}$denotes the electron operator on the top layer and $c_{-}$the electron operator on the bottom layer.) Now, let us consider the following interchain electron pairing and backscattering term. Combining Eqs. (22) and (23) yields the following transmutation:

$$
-g_{I, I+1} \operatorname{Re}\left(c_{-R}^{I \dagger}-c_{+R}^{I}\right)\left(c_{-L}^{I+1}-c_{+L}^{I+1 \dagger}\right) \propto-g_{I, I+1}^{*} \epsilon_{R}^{I} \epsilon_{L}^{I+1},
$$

where $g_{I, I+1}^{*}=g_{I, I+1}\left\langle\cos \left(\phi_{s, R}^{I} / \nu_{s}-\phi_{s, L}^{I+1} / \nu_{s}\right)\right\rangle$. Therefore, the interchain electron pairing and backscattering can be added to the Hamiltonian of $Z_{3}$ parafermions to couple neighboring chains in the above fashion. It is known that for the $Z_{3}$ parafermion CFT, perturbing the Hamiltonian with a thermal operator moves the theory off the critical point for all values of $g_{I, I+1}^{*}$ [56]. However, $g_{I, I+1}^{*}>0$ drives the system into a paramagnetic phase, while $g_{I, I+1}^{*}<0$ to the ferromagnetic phase.

Consequently, if we add the above interchain mass term to the array of gapless $Z_{3}$ parafermion chains, we can gap out all the gapless modes except for the two outermost ones. Next, we appeal to the bulk-boundary conjecture to relate the topological order of the bulk to its edge CFT. Since the resulting FTSC and Read-Rezayi $Z_{3}$ parafermion states of the $\nu_{c}=13 / 5 \mathrm{FQH}$ are both gapped in their bulks and have identical non-Abelian edge CFT, therefore, they are described by the same non-Abelian topological order and support the same non-Abelian excitations. Among these excitations, we find that the most significant one is the 
Fibonacci anyon that is capable of performing topological quantum computation via braiding operations.

Now, let us imagine an FTSC whose parent state is at the filling fraction $\nu_{c}=2 / 3$. The value of $k$ in the sineGordon model is $k=2 / \nu_{c}=3$. Hence, we obtain the $Z_{3}$ parafermion FTSC by inducing superconductivity into the $\nu_{c}=2 / 3 \mathrm{FQH}$ state. Two fermionic candidates for such a state are the $(3,3,0),(2,2,1)$, and $(1,1,2)$ Halperin states. These bilayer FQH states have neutral and charged degrees of freedom, each described by $c=1$ CFT. Superconductivity cannot do anything with the neutral part, and only the charged part undergoes the phase transition into the $Z_{3}$ parafermion state. However, we must gap out these neutral sectors first in order to have an excitation with multiples of the $q=2 / 3$ electric charge. Thus, the $\nu_{c}=$ $2 / 3 \mathrm{FQH}-\mathrm{SC}$ heterostructure can yield the superconducting analogue of the $Z_{3}$ parafermion $\mathrm{FQH}$ state.

\section{PARAFERMION FRACTIONAL TOPOLOGICAL SUPERCONDUCTORS}

In this section, we apply the Teo-Kane construction [48] to obtain a $2 \mathrm{D}$ topological state from an array of $1 \mathrm{D}$ gapless chains. To this end, we couple the 1D parafermion chains to one another (see Fig. 3). We first map the 2D FCI at the $\nu_{c}=2 / k$ filling fraction into an array of 1D chains of free bosons. When spin-orbit coupling is strong in the parent state, we can generate intrachain electron backscattering through proximity to a ferromagnet. On the other hand, the superconducting substrate generates intrachain electron pairing. Therefore, each chain is described by the SDSG model in Eq. (6). Let us call the intrachain perturbations coming from these two processes $T_{1}^{I, I}$. At the self-dual point, i.e., when $g_{1}=g_{2}$, the perturbations drive a phase transition from the UV fixed point with $c_{\mathrm{UV}}=1$ into the IR fixed point that is now described by $Z_{k}$ parafermion theory with $c_{\mathrm{IR}}=2(k-1) /(k+2)$ for $k<4$. Tuning the interaction to the self-dual point is a stringent condition. Deviation from this condition will be addressed shortly.
Then, we study the effect of intrachain anyon and electron backscattering along with electron pairing. Let us call this interchain perturbation $T_{2}^{I, I+1}$. In Sec. III, we checked that for the $k=2,3$ cases, these terms can be rewritten in terms of the primary fields of the emergent $Z_{k}$ CFT. We see that they correspond to coupling the holomorphic operators on the Ith chain to the antiholomorphic operator on the $(I+1)$ th chain. Such perturbations gap out both gapless modes that are involved. Therefore, by applying the $T_{2}^{I, I+1}$ terms, we can gap out all the parafermion modes in the system except for the two outermost ones: the left-moving parafermion mode at the first chain and the right-moving parafermion mode at the last chain. Altogether, we obtain a gapped bulk that has a chiral parafermion edge state. Bulkboundary CFT correspondence implies that all states with the same edge CFT have the same operator content and identical topological orders. Therefore, the state we find is the superconducting analogue of the (non-Abelian part of the) Read-Rezayi parafermion states originally proposed for the $\mathrm{FQH}$ systems.

Now, let us discuss the effect of moving away from the self-dual point of the SDSG model in Eq. (6). Since the 2D $Z_{k}$ parafermion state that we obtain by starting from the self-dual point $\left(g_{1}=g_{2}\right)$ is gapped, it is robust against perturbations and maintains its topological properties as long as perturbations do not close the gap. The bulk gap is determined by the interchain interaction $T_{2}^{I, I+1}$. Assuming that it opens a mass gap $\Delta$, we expect the system to stay in the $Z_{k}$ parafermion state as long as the distance from the self-dual point, which is proportional to $\left|g_{1}-g_{2}\right|$, does not exceed $\Delta$. Therefore, the exact self-duality condition is not necessary.

We would like to emphasize again that the above picture applies to $k=2 / \nu_{c}<4$ cases only, i.e., when $\nu_{c} \in\{1,2 / 3\}$. For other values of $k$, the cosine terms in Eq. (6) are irrelevant and do not drastically modify the lowenergy physics. However, our simple RG analysis may fail for other cases and we may obtain $Z_{k}$ parafermion FTSCs in other cases as well. One reason is that our RG analysis is
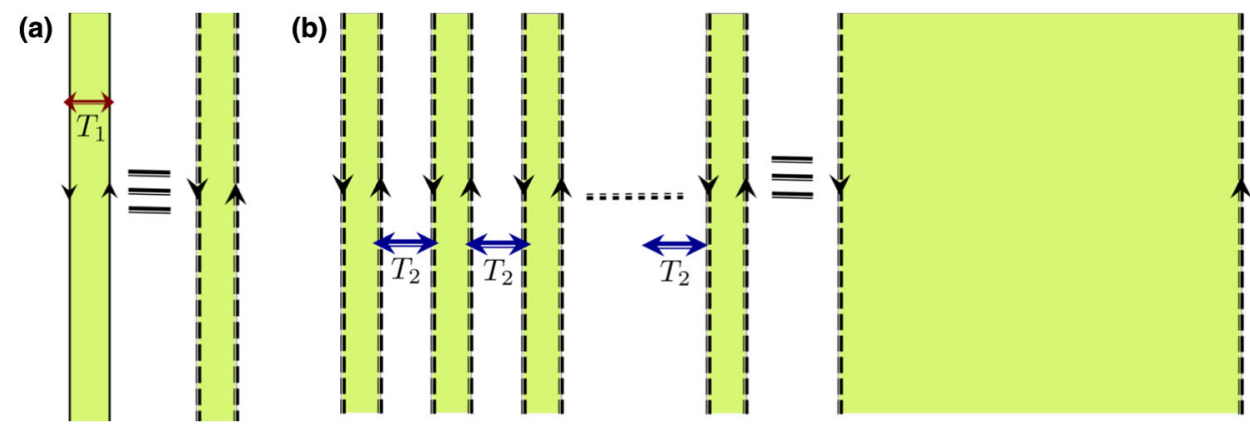

FIG. 3. Array of coupled $1 \mathrm{D}$ chain. (a) $T_{1}$ represents the intrachain interaction that turns a chain into a parafermion chain. The solid (dashed) line denotes a free-boson (parafermion) gapless mode. (b) $T_{2}$ represents the interchain coupling that gaps out every two adjacent parafermion modes. After applying the $T_{1}$ and $T_{2}$ terms, we are left with a gapped bulk and a chiral gapless parafermion edge mode. 
based on 1D CFT calculation, while the system that we have in hand is, in fact, a 2D system. If we believe in the RG analysis based on the 1D CFT, the low-energy physics (the IR limit) of the SDSG is described by a $c=1$ CFT for $k>4$. We conjecture that this theory is a $U(1)_{k} / Z_{2}$ orbifold CFT. The unperturbed Hamiltonian of the 1D chains in Eq. (6) (i.e., when $g_{1}=g_{2}=0$ ) is described by a $U(1)_{k}$ CFT of free bosons. In this case, the chiral algebra is generated by the current operator $j=\partial \phi$ and creation $c_{R(L)}^{\dagger}$ and annihilation $c_{R(L)}$ operators, where $c_{R(L)} \sim e^{i k \phi_{R(L)}}$. However, superconductivity breaks the $U(1)$ symmetry associated with the charge degree of freedom. So, we cannot have both $c_{R(L)}$ and $c_{R(L)}^{\dagger}$ in the chiral algebra. One possibility is that perturbation makes the $i\left(c_{R(L)}-c_{R(L)}^{\dagger}\right) \sim$ $\sin \left(k \phi_{R(L)}\right)$ operator gapped while it maintains the other combination $c_{R(L)}+c_{R(L)}^{\dagger} \sim \cos \left(k \phi_{R(L)}\right)$ as gapless. In this case, the chiral algebra is generated by the current operator $j$ and $\cos \left(k \phi_{R(L)}\right)$ operators only. The latter operator is invariant under the following discrete $Z_{2}$ symmetry: $\phi_{R(L)} \rightarrow-\phi_{R(L)}$. Therefore, we can identify $\phi_{R(L)}$ with $-\phi_{R(L)}$. The resulting chiral algebra is well studied and gives rise to the $U(1)_{k} / Z_{2}$ orbifold CFT. Interestingly, this theory supports twist operators with quantum dimensions equal to $d_{\mathrm{tw}}=\sqrt{k}$ that are physical manifestations of the bulk superconducting vortices; namely, the electron operator picks a negative sign when it circles around twist operators [9]. Therefore, we conjecture that an FTSC whose parent state is at the $\nu_{c}=2 / k<1 / 2$ filling fraction is described by a $U(1)_{k} / Z_{2}$ orbifold CFT instead of a $Z_{k}$ parafermion theory.

\section{SUMMARY AND CONCLUSION}

In this paper, we explained that a fractional quantum Hall system at the $\nu_{c}=2 / k$ filling fraction can be mapped to an array of narrow strips, each with a pair of counterpropagating gapless modes. These narrow strips can be viewed as quasi-1D chains whose charged sectors are described by a $U(1)$ CFT. The coupling between the right-moving branch of the Ith chain and the left-moving branch of the $(I+1)$ th chain gaps out the bulk but maintains the edge modes of the $2 \mathrm{D}$ system as gapless. If we assume spin-orbit coupling is so large in the system that the spin of the electron is locked to its momentum and that, as a result, all right- (left-) moving sectors carry spin up (down), then we can induce $s$-wave paring as well as in-plane magnetic order in the bulk of the system via proximity or intrinsic spontaneous symmetry breaking.

By inducing superconductivity in the bulk of a fractional quantum Hall state, we can obtain other possibilities besides the above-mentioned mechanism for achieving the Abelian phase from coupled 1D chains. As we discussed in the current paper, we can first couple the right- and left-moving parts of each chain (intrachain interaction) and tune the strength of pairing and backscattering to obtain gapless $Z_{k}$ parafermion chains. Then, adjacent parafermion modes can be coupled to gap out the bulk of the system. The edge of the system is gapless, chiral, and is described by a $Z_{k}$ parafermion CFT. We can understand this result in a more intuitive way. The electron operator in the $\nu_{c}=2 / k \mathrm{FQH}$ state is equivalent to $k / 2$ anyons (when all neutral fluctuations are gapped). Accordingly, a Cooper pair consists of $k$ anyons. As a result, we expect a topological state with $k$-anyon condensation to emerge through the proximity effect. One candidate for such a condensate is the Read-Rezayi $Z_{k}$ parafermion state. The most interesting case is a $\nu_{c}=2 / 3$ $\mathrm{FQH}$ on top of a superconducting substrate. By increasing the strength of pairing, the system undergoes a topological phase transition into the $Z_{3}$ parafermion FTSC. The operator content of this state contains Fibonacci anyons that are capable of performing universal quantum computation. The $\nu_{c}=2 / 3 \mathrm{FQH}$ can be achieved in $(3,3,0)$, $(2,2,1)$, or $(1,1,2)$ bilayer states. For the $(3,3,0)$ bilayer state, we need to add an interlayer pairing; otherwise, the two layers would act independently and would have $\nu_{\uparrow}=\nu_{\downarrow}=1 / 3$ effective fillings.

\section{ACKNOWLEDGEMENTS}

We gratefully acknowledge useful discussions with E.-A. Kim, T. Senthil, M.-Z. Hasan, N. Regnault, S. Raghu, and S.-B. Chung. We are, in particular, thankful to Maissam Barkeshli for his insightful comments and valuable discussions. This work was supported by NSF CAREER Grant No. DMR-0955822.

Note added.-During the completion of this work, we became aware of, through arXiv posting, another independently carried-out work on a related but different topological order for the $2 / 3 \mathrm{FQH}-\mathrm{SC}$ heterostructure setup, Ref. [63].

[1] C. Nayak, S. H. Simon, A. Stern, M. Freedman, and S. Das Sarma, Non-Abelian Anyons and Topological Quantum Computation, Rev. Mod. Phys. 80, 1083 (2008).

[2] M. Zahid Hasan and C. L. Kane, Colloquium: Topological Insulators, Rev. Mod. Phys. 82, 3045 (2010).

[3] X.-L. Qi and S.-C. Zhang, Topological Insulators and Superconductors, Rev. Mod. Phys. 83, 1057 (2011).

[4] X.-G. Wen, Non-Abelian Statistics in the Fractional Quantum Hall States, Phys. Rev. Lett. 66, 802 (1991).

[5] G. Moore and N. Read, Nonabelions in the Fractional Quantum Hall Effect, Nucl. Phys. B360, 362 (1991).

[6] N. H. Lindner, E. Berg, G. Refael, and A. Stern, Fractionalizing Majorana Fermions: Non-Abelian Statistics on the Edges of Abelian Quantum Hall States, Phys. Rev. X 2, 041002 (2012). 
[7] D. J. Clarke, J. Alicea, and K. Shtengel, Exotic Non-Abelian Anyons from Conventional Fractional Quantum Hall States, Nat. Commun. 4, 1348 (2013).

[8] M. Cheng, Superconducting Proximity Effect on the Edge of Fractional Topological Insulators, Phys. Rev. B 86, 195126 (2012).

[9] A. Vaezi, Fractional Topological Superconductor with Fractionalized Majorana Fermions, Phys. Rev. B 87, 035132 (2013).

[10] N. Read and E. H. Rezayi, Beyond Paired Quantum Hall States: Parafermions and Incompressible States in the First Excited Landau Level, Phys. Rev. B 59, 8084 (1999).

[11] P. Di Francesco, P. Mathieu, and D. Senechal, Conformal Field Theory (Springer, New York, 1996).

[12] N. Read and D. Green, Paired States of Fermions in Two Dimensions with Breaking of Parity and Time-Reversal Symmetries and the Fractional Quantum Hall Effect, Phys. Rev. B 61, 10267 (2000).

[13] D. A. Ivanov, Non-Abelian Statistics of Half-Quantum Vortices in p-Wave Superconductors, Phys. Rev. Lett. 86, 268 (2001).

[14] M. H. Freedman, M. J. Larsen, and Z. Wang, A Modular Functor Which Is Universal for Quantum Computation, Commun. Math. Phys. 227, 605 (2002).

[15] N. E. Bonesteel, L. Hormozi, G. Zikos, and S. H. Simon, Braid Topologies for Quantum Computation, Phys. Rev. Lett. 95, 140503 (2005).

[16] L. Hormozi, G. Zikos, N. E. Bonesteel, and S. H. Simon, Topological Quantum Compiling, Phys. Rev. B 75, 165310 (2007).

[17] E. Fradkin and L. P. Kadanoff, Disorder Variables and Parafermions in Two-Dimensional Statistical Mechanics, Nucl. Phys. B170, 1 (1980).

[18] Z. Nussinov, G. Ortiz, and E. Cobanera, Arbitrary Dimensional Majorana Dualities and Architectures for Topological Matter, Phys. Rev. B 86, 085415 (2012).

[19] P. Fendley, Parafermionic Edge Zero Modes in Zn-Invariant Spin Chains, J. Stat. Mech. (2012) P11020.

[20] E. Cobanera and G. Ortiz, Fock Parafermions and Self-Dual Representations of the Braid Group, arXiv:1307.6214.

[21] M. Levin and A. Stern, Fractional Topological Insulators, Phys. Rev. Lett. 103, 196803 (2009).

[22] S. Beigi, P. W. Shor, and D. Whalen, The Quantum Double Model with Boundary: Condensations and Symmetries, Commun. Math. Phys. 306, 663 (2011).

[23] M. B. Hastings, C. Nayak, and Z. Wang, Metaplectic Anyons, Majorana Zero Modes, and Their Computational Power, Phys. Rev. B 87, 165421 (2013).

[24] J. Motruk, E. Berg, A. M. Turner, and F. Pollmann, Topological Phases in Gapped Edges of Fractionalized Systems, Phys. Rev. B 88, 085115 (2013).

[25] R. Bondesan and T. Quella, Topological and Symmetry Broken Phases of $Z_{N}$ Parafermions in One Dimension, J. Stat. Mech. (2013) P10024.

[26] M. Burrello, B. van Heck, and A. R. Akhmerov, Braiding of Non-Abelian Anyons Using Pairwise Interactions, Phys. Rev. A 87, 022343 (2013).

[27] P. Bonderson, Measurement-Only Topological Quantum Computation via Tunable Interactions, Phys. Rev. B 87, 035113 (2013).
[28] H. Bombin, Topological Order with a Twist: Ising Anyons from an Abelian Model, Phys. Rev. Lett. 105, 030403 (2010).

[29] M. Barkeshli and X.-L. Qi, Topological Nematic States and Non-Abelian Lattice Dislocations, Phys. Rev. X 2, 031013 (2012).

[30] M. Barkeshli, C.-M. Jian, and X.-L. Qi, Twist Defects and Projective Non-Abelian Braiding Statistics, Phys. Rev. B 87, 045130 (2013).

[31] M. Burrello, B. van Heck, and E. Cobanera, Topological Phases in Two-Dimensional Arrays of Parafermionic Zero Modes, Phys. Rev. B 87, 195422 (2013).

[32] Y.-Z. You and X.-G. Wen, Projective Non-Abelian Statistics of Dislocation Defects in a $Z_{N}$ Rotor Model, Phys. Rev. B 86, 161107 (2012).

[33] M. Barkeshli and X.-L. Qi, Synthetic Topological Qubits in Conventional Bilayer Quantum Hall Systems, arXiv:1302.2673.

[34] M. Barkeshli, C.-M. Jian, and X.-L. Qi, Classification of Topological Defects in Abelian Topological States, arXiv:1304.7579.

[35] J. C. Y. Teo, A. Roy, and X. Chen, Unconventional Fusion and Braiding of Topological Defects in a Lattice Model, arXiv:1306.1538.

[36] E. Tang, J.-W. Mei, and X.-G. Wen, High-Temperature Fractional Quantum Hall States, Phys. Rev. Lett. 106, 236802 (2011).

[37] T. Neupert, L. Santos, C. Chamon, and C. Mudry, Fractional Quantum Hall States at Zero Magnetic Field, Phys. Rev. Lett. 106, 236804 (2011).

[38] D. N. Sheng, Z.-C. Gu, K. Sun, and L. Sheng, Fractional Quantum Hall Effect in the Absence of Landau Levels, Nat. Commun. 2, 389 (2011).

[39] K. Sun, Z. Gu, H. Katsura, and S. Das Sarma, Nearly Flatbands with Nontrivial Topology, Phys. Rev. Lett. 106, 236803 (2011).

[40] N. Regnault and B. Andrei Bernevig, Fractional Chern Insulator, Phys. Rev. X 1, 021014 (2011).

[41] Y.-L. Wu, B. Andrei Bernevig, and N. Regnault, Zoology of Fractional Chern Insulators, Phys. Rev. B 85, 075116 (2012).

[42] A. Vaezi, Fractional Quantum Hall Effect at Zero Magnetic Field, arXiv:1105.0406.

[43] P. Lecheminant, A. O. Gogolin, and A. A. Nersesyan, Criticality in Self-Dual Sine-Gordon Models, Nucl. Phys. B639, 502 (2002).

[44] P. Lecheminant and E. Orignac, $Z_{3}$ Quantum Criticality in a Spin-1/2 Chain Model, Phys. Rev. B 69, 174409 (2004).

[45] R. Dijkgraaf, C. Vafa, E. Verlinde, and H. Verlinde, The Operator Algebra of Orbifold Models, Commun. Math. Phys. 123, 485 (1989).

[46] M. Barkeshli and X.-G. Wen, Anyon Condensation and Continuous Topological Phase Transitions in Non-Abelian Fractional Quantum Hall States, Phys. Rev. Lett. 105, 216804 (2010).

[47] M. Barkeshli and X.-G. Wen, Bilayer Quantum Hall Phase Transitions and the Orbifold Non-Abelian Fractional Quantum Hall States, Phys. Rev. B 84, 115121 (2011). 
[48] J. C. Y. Teo and C. L. Kane, From Luttinger Liquid to NonAbelian Quantum Hall States, arXiv:1111.2617.

[49] X.-G. Wen, Quantum Field Theory of Many-Body Systems from the Origin of Sound to an Origin of Light and Electrons (Oxford University Press, New York, 2004).

[50] Y.-M. Lu and A. Vishwanath, Theory and Classification of Interacting Integer Topological Phases in Two Dimensions: A Chern-Simons Approach, Phys. Rev. B 86, 125119 (2012).

[51] J. Wang and X.-G. Wen, Boundary Degeneracy of Topological Order, arXiv:1212.4863.

[52] It should be emphasized again that in the case of multicomponent (multilayer) FQH states, $e^{i \phi_{R(L)} / \nu_{c}}$ only represents the charged part of the electron operator. The neutral part is described by other bosonic degrees of freedom that are not affected by superconducting pairing. We assume all neutral sectors are gapped.

[53] A. Vaezi and E.-A. Kim, UV-IR Transmutation for Hybrid Realizations of $Z_{k}$ Parafermion Systems, arXiv:1310.7434.

[54] A. B. Zamolodchikov, O Neobratimosti Potoka Renormalizatsionnoi Gruppy v Dvumernoi Teorii Polya, Pis'ma Zh. Eksp. Teor. Fiz. 43, 565 (1986) [Irreversibility of the Flux of the Renormalization Group in a 2D Field Theory, JETP Lett. 43, 730 (1986)].

[55] J. V. Jose, L. P. Kadanoff, S. Kirkpatrick, and D. R. Nelson, Renormalization, Vortices, and Symmetry-Breaking Perturbations in the Two-Dimensional Planar Model, Phys. Rev. B 16, 1217 (1977).
[56] P. Dorey, R. Tateo, and K. E. Thompson, Massive and Massless Phases in Self-Dual ZN Spin Models: Some Exact Results from the Thermodynamic Bethe Ansatz, Nucl. Phys. B470, 317 (1996).

[57] D. G. Shelton, A. A. Nersesyan, and A. M. Tsvelik, Antiferromagnetic Spin Ladders: Crossover between Spin $S=1 / 2$ and $S=1$ Chains, Phys. Rev. B 53, 8521 (1996).

[58] L. Fu and C. L. Kane, Superconducting Proximity Effect and Majorana Fermions at the Surface of a Topological Insulator, Phys. Rev. Lett. 100, 096407 (2008).

[59] X.-L. Qi, T. L. Hughes, and S. Zhang, Chiral Topological Superconductor from the Quantum Hall State, Phys. Rev. B 82, 184516 (2010).

[60] V. A. Fateev and Al. B. Zamolodchikov, Integrable Perturbations of $Z_{N}$ Parafermion Models and the O(3) Sigma Model, Phys. Lett. B 271, 91 (1991).

[61] P. Baseilhac and V. A. Fateev, Expectation Values of Local Fields for a Two-Parameter Family of Integrable Models and Related Perturbed Conformal Field Theories, Nucl. Phys. B532, 567 (1998).

[62] V. A. Fateev, Normalization Factors in Conformal Field Theory and Their Applications, Mod. Phys. Lett. A 15, 259 (2000).

[63] R. S. K. Mong, D. J. Clarke, J. Alicea, N. H. Lindner, P. Fendley, C. Nayak, Y. Oreg et al., Universal Topological Quantum Computation from a Superconductor-Abelian Quantum Hall Heterostructure, Phys. Rev. X 4, 011036 (2014). 